\title{
A Swedish heterodyne facility instrument for the APEX telescope
}

\author{
V. Vassilev ${ }^{1}$, D. Meledin ${ }^{1}$, I. Lapkin ${ }^{1}$, V. Belitsky ${ }^{1}$, O. Nyström ${ }^{1}$, D. Henke ${ }^{1}$, A. Pavolotsky ${ }^{1}$, R. Monje ${ }^{1}$, C. Risacher ${ }^{2}$, \\ M. Olberg ${ }^{1}$, M. Strandberg ${ }^{1}$, E. Sundin ${ }^{1}$, M. Fredrixon ${ }^{1}$, S.-E. Ferm ${ }^{1}$, V. Desmaris ${ }^{1}$, D. Dochev ${ }^{1}$, \\ M. Pantaleev ${ }^{1}$, P. Bergman ${ }^{1,2}$, and H. Olofsson ${ }^{1}$ \\ 1 Onsala Space Observatory (OSO), Chalmers University of Technology, 43992, Sweden \\ e-mail: vessen.vassilev@chalmers.se \\ 2 European Southern Observatory (ESO), Casilla 19001, Santiago 19, Chile
}

Received 25 June 2008 / Accepted 25 August 2008

\section{ABSTRACT}

\begin{abstract}
Aims. In March 2008, the APEX facility instrument was installed on the telescope at the site of Lliano Chajnantor in northern Chile. The main objective of the paper is to introduce the new instrument to the radio astronomical community. It describes the hardware configuration and presents some initial results from the on-sky commissioning.

Methods. The heterodyne instrument covers frequencies between $211 \mathrm{GHz}$ and $1390 \mathrm{GHz}$ divided into four bands. The first three bands are sideband-separating mixers operating in a single sideband mode and based on superconductor-insulator-superconductor (SIS) tunnel junctions. The fourth band is a hot-electron bolometer, waveguide balanced mixer. All bands are integrated in a closedcycle temperature-stabilized cryostat and are cooled to $4 \mathrm{~K}$.

Results. We present results from noise temperature, sideband separation ratios, beam, and stability measurements performed on the telescope as a part of the receiver technical commissioning. Examples of broad extragalactic lines are also included.
\end{abstract}

Key words. telescopes - instrumentation: detectors - techniques: spectroscopic - radio lines: general

\section{Introduction}

The Atacama Pathfinder Experiment (APEX) is a collaboration between the Max Planck Institute for Radio Astronomy, Onsala Space Observatory (OSO), and the European Southern Observatory (ESO) (Güsten et al. 2006a). APEX is a $12 \mathrm{~m}$ single-dish telescope with a surface accuracy of $17 \mu \mathrm{m}$ (rms), allowing observations in the sub-mm region (up to $1600 \mathrm{GHz}$ ).

The Onsala Space Observatory is committed to delivering to the telescope a heterodyne single-pixel instrument (Belitsky et al. 2007), covering frequencies between 211 and $1390 \mathrm{GHz}$ divided into four bands, which are summarized in Table 1. The Swedish Heterodyne Facility Instrument (SHFI) is a replacement for the APEX 2A receiver (Risacher et al. 2006b) installed in 2005. The first three bands are based on SIS tunnel junctions and take advantage of the sideband-separating mixer technology previously developed at OSO. Band T2 is a hot-electron bolometer (HEB), waveguide, balanced mixer.

This paper summarizes the results of the technical commissioning of bands 1, 2, and T2, which took place in March 2008. The rejection ratios were verified using a harmonic mixer to generate a test signal at the corresponding sidebands. The measurement was repeated using on-sky sources. The rejection ratios were found to be better in the case of on-sky sources.

Beam shapes and sizes were also verified using continuum scans of planets. At the end of the paper, we present some examples of extragalactic spectra taken with different bands of the instrument.

\section{Instrument configuration}

The SHFI is located in the Nasmith cabin A of the telescope. The cryostat of the receiver (Belitsky et al. 2006) is designed
Table 1. SHFI frequency bands.

\begin{tabular}{cccc}
\hline \hline $\begin{array}{c}\text { APEX } \\
\text { Band }\end{array}$ & $\begin{array}{c}\text { Frequency } \\
\text { GHz }\end{array}$ & Technology & $\begin{array}{c}H P B W \\
\operatorname{arcsec}\end{array}$ \\
\hline 1 & $211-275$ & SIS, 2SB & $30-23$ \\
2 & $275-370$ & SIS, 2SB & $23-17$ \\
$3^{*}$ & $375-500$ & SIS, 2SB & $17-13$ \\
T2 & $1250-1390$ & HEB Balanced, Waveguide & 5 \\
\hline
\end{tabular}

${ }^{*}$ Band 3 is not yet installed in the cryostat.

to accommodate six frequency bands and the top plate therefore contains 12 optical windows (6 RF and $6 \mathrm{LO}$ for each of the six channels). A schematic of the instrument is shown in Fig. 1. The beam is directed from the Cassegrain cabin and through a flange enters cabin A. The flange is also used as a reference plane for the optical alignment. After entering the cabin, the beam is reflected by an instrument-switching mirror, which switches the beam between SHFI and a second (PI) instrument.

The implemented optical solution (Lapkin et al. 2008) allows observation with one frequency band of SHFI at a given time. To select the required band, an active, channel-switching, mirror reflects the beam into a specific channel mirror directing the beam to the corresponding window of the cryostat. The top plate of the cryostat also accommodates all LO sources that follow a separate optical path (see the insert of Fig. 1). All LO sources are direct multiplication chains using a commercial synthesizer as an input signal. The LO power is adjusted by a control voltage applied to an amplifier that is a part of the chain. To provide better stability and also shorter switching times between the bands, all LO sources are temperature stabilized. 


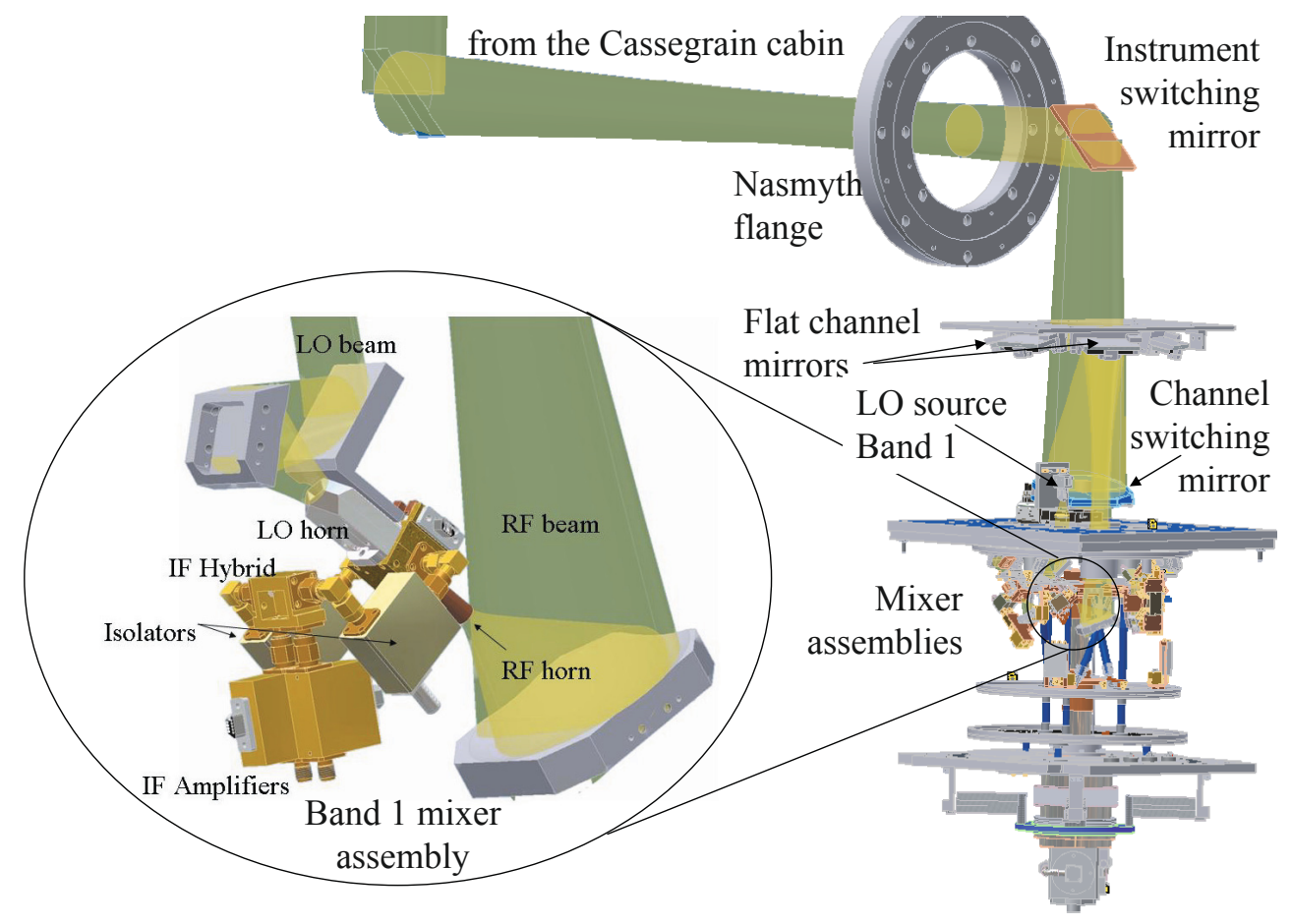

Fig. 1. Structure of the SHFI cryostat.

The cryostat is based on a three-stage cryo-cooler (model SRDK-3ST) produced by Sumitomo Heavy Industries. Unloaded, the final stage provides temperature of about $3.6 \mathrm{~K}$ and is equipped with a liquid $\mathrm{He}$ pot for temperature stabilization. To protect the RF parts of the receiver from vibrations, the cryo-cooler is mechanically isolated from the dewar by suspending it on vibration dampers. In addition, all thermal connections between the cryo-cooler and the mixer assemblies are provided by flexible links made of oxygen-free copper wires.

\section{SHFI bands}

We briefly describe the hardware and present some noise, stability, and sideband separation measurements performed after the installation on the telescope. We also provide references to papers where mixers designs are described in detail.

\subsection{Band 1}

The band 1 2SB mixer assembly, shown in Fig. 1, is based on a compact design in which all mixer components (except IF hybrid and isolators) are integrated onto a single mixer block. The RF and LO beams enter at opposite sides of the mixer block. A pair of isolators connect the IF outputs to the hybrid where the sideband cancelation takes place. Both sidebands are available for amplification at the outputs of the hybrid. To reduce the size of the 2SB mixer assembly, and to minimize losses, we developed a superconducting IF hybrid using the same thin $\mathrm{Nb}$ process as for the mixer fabrication. The width of the hybrid is adjusted so that it fits the mixer block shown in the insert of Fig. 1. The same approach is used in the design of band 2.

Figure 2 illustrates the structure of the mixer block. The $\mathrm{RF}$ is applied to a waveguide, branch-line, coupler, the divided RF power is coupled to the mixer substrate by two radial E-probes at the ends of the mixer chip. The LO power is applied to the middle of the mixer chip, where a double-probe

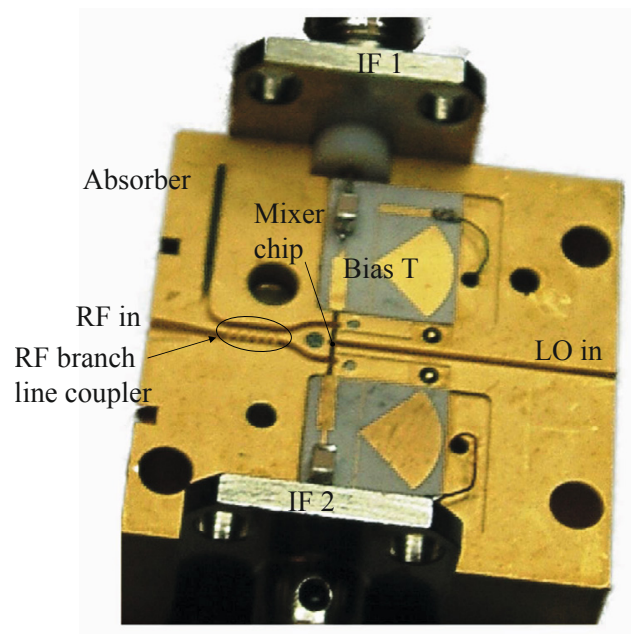

Fig. 2. Band 1 mixer block.

structure (Vassilev et al. 2001) divides the LO power and provides the transition from waveguide to microstrip line. To couple LO power to the junctions, we developed an on-chip directional coupler and a broadband termination based on thin-film microstrip lines (Vassilev et al. 2008; Monje et al. 2004). The mixer circuitry is based on SIS twin-junctions, where the two junctions are located at the ends of a transmission line so that their reactance is mutually compensated at RF. Each SIS junction has an area of $4 \mu \mathrm{m}^{2}$ and a normal state resistance of $7.5 \Omega$. This combination of SIS area, resistance, and tuning circuitry provides a broad RF range, while the relatively large junction area facilitates the use of optical lithography. Two bias-Ts, integrated into the mixer block, are used to extract the IF power, and also provide biasing and the appropriate IF embedding impedance for the SIS junctions (Fig. 2). After sideband cancelation in the IF hybrid and amplification, the separated sidebands are accessible at the outputs of the amplifiers. 

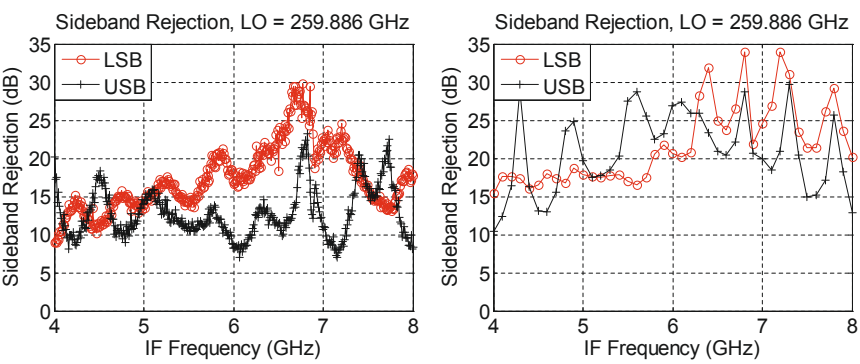

Fig. 3. SRR measured with two amplifiers (left) and with termination replacing one of the amplifiers.

During the laboratory tests, we realized that the rejection ratio could be improved significantly if one of the IF amplifiers was removed and replaced by a matched load (in this case only one sideband was available at the time). The cryogenic, lownoise, IF amplifiers (Risacher et al. 2003) have a relatively high reflection coefficient at their inputs, whereas the load provides higher quality termination for the hybrid ensuring better rejection ratios with less variations across the IF band. The SRR improvement was experimentally confirmed and observed at many LO frequencies, for both band 1 and 2 . Figure 3 provides an example of the SRR measured in the case of two IF amplifiers, and one amplifier and one matched load. We allowed band 1 and 2 mixers to operate in SSB instead of 2SB mode, because of the SRR improvement. At a fixed LO frequency, the desired sideband was chosen by biasing the mixers: the positive-bias voltages produced USB, whereas negative-bias voltage on one of the mixers produced LSB. After its installation at the telescope, noise and sideband rejection measurements vsersus IF were performed for a single LO frequency to confirm that the performance was consistent with previous laboratory tests. The noise temperature and SRR were measured at the RF window using hot/cold absorbers and a harmonic mixer as a source of test signal; the results of this test are shown in Fig. 4. The noise measurement agrees with that obtained in the laboratory tests, while the SRR were better by $\sim 5 \mathrm{~dB}$ when measured at the telescope. The reason for this improvement is related to the power and spectral content of the test source causing mixer saturation and/or its optical alignment.

The noise temperature across the RF band was verified at the telescope using an additional pair of mirrors that directed the beam to a facility calibration unit providing hot/cold loads. Figure 5 shows the averaged SSB noise temperatures of each sideband for $1 \mathrm{GHz}$ of bandwidth between 5 and $6 \mathrm{GHz}$. For a specific RF frequency, the program that tunes the receiver, checks the frequency points in a tuning table and selects the sideband that provides a lower noise temperature.

\subsection{Band 2}

Band 2 is based on a modular type of design in which two DSB mixers are connected to a middle piece that contains the RF and LO distribution circuitry (Fig. 6). The middle piece divides the RF/LO signals and couples LO to the RF paths with the appropriate phasing (Risacher et al. 2006a; Monje et al. 2006). As in the case of Band 1, the noise temperature and SRR were measured at the RF window for a single LO frequency. The results of this test versus IF is shown in Fig. 7. The noise measurement is consistent with the laboratory tests while the SRR is again better.

The noise temperature across the RF band was verified after installation using the facility calibration unit for hot/cold loads.

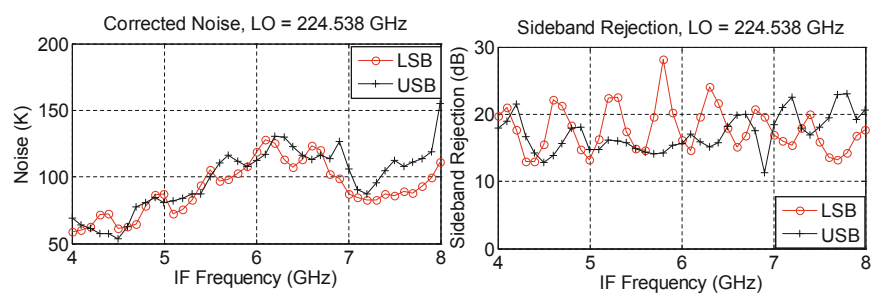

Fig. 4. Band 1 after the installation on the telescope. The SSB noise temperature for both sidebands (left) and the SRR (right).

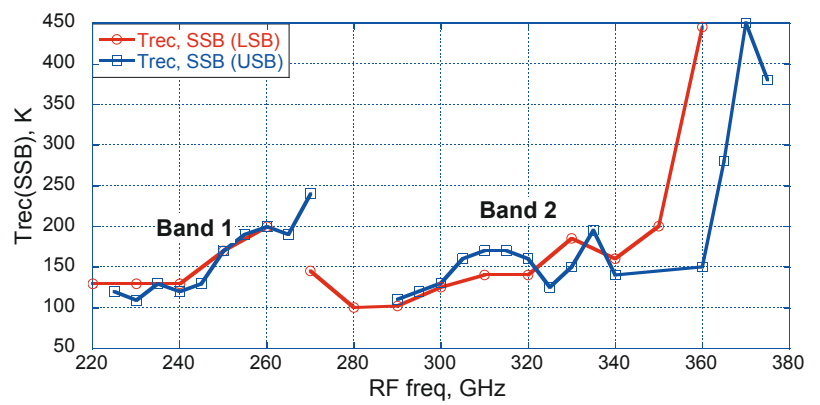

Fig. 5. Band 1, 2 SSB noise temperatures for both sidebands measured with the facility calibration unit.

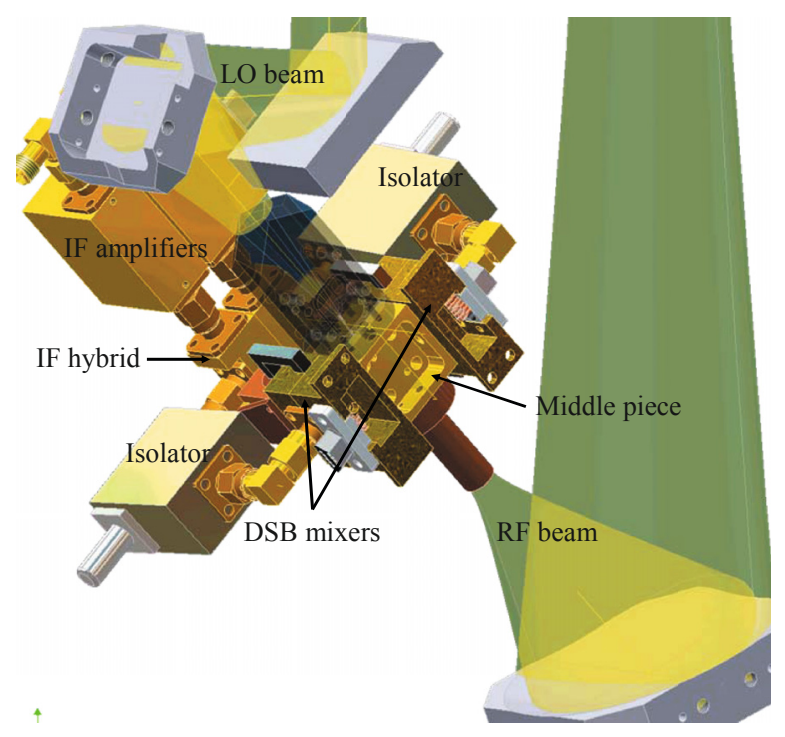

Fig. 6. Band 2 mixer assembly.

Figure 5 shows the averaged noise temperature for $1 \mathrm{GHz}$ of bandwidth between 5 and $6 \mathrm{GHz}$ and for both sidebands.

\subsection{Band T2}

Band T2 DSB receiver covers a $1250-1390 \mathrm{GHz}$ frequency range and employs a waveguide balanced scheme (Meledin et al. 2006, 2008). The balanced scheme has a number of benefits over single-ended mixers. Among them are the suppression of LO amplitude modulation noise, better LO power-handling capabilities, and rejection of unwanted spurious responses (Maas 1993; Kerr et al. 1996). On the other hand, the high operating frequencies, above $1 \mathrm{THz}$, introduce significant difficulties for manufacturing waveguide components, and make balanced design less attractive. 

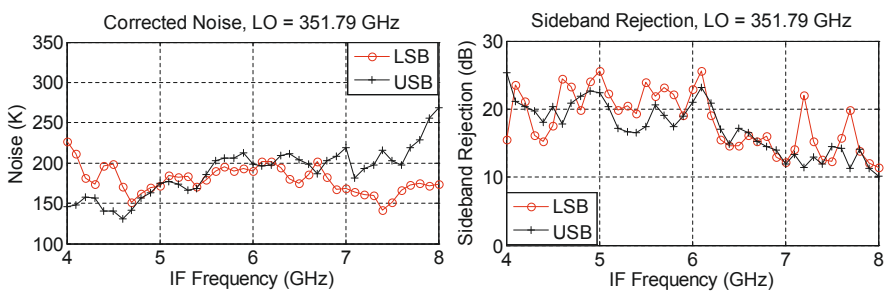

Fig. 7. Band 2 after the installation on the telescope. The SSB noise temperature for both sidebands (left) and the SRR (right).

Band T2 is based on a quadrature scheme consisting of a $3 \mathrm{~dB} 90^{\circ}$ waveguide RF hybrid, two identical superconducting hot-electron bolometer (HEB) mixers, and a commercial $180^{\circ}$ IF hybrid. The RF hybrid provides the LO and RF signal distribution between individual HEB mixers and consists of two parallel waveguides with dimensions of $90 \mu \mathrm{m} \times 180 \mu \mathrm{mm}$ coupled through 6 branches between the broad walls. The optimized minimum aperture's height and length are as small as $21 \mu \mathrm{m}$ and $50 \mu \mathrm{m}$, respectively. To ensure the required amplitude and phase balance, the hybrid must be fabricated with a linear error less than $2 \mu \mathrm{m}$. For fabrication of the hybrid, we employed photolithography of thick photoresist combined with fine electroplating described in detail in Pavolotsky et al. (2005) and Desmaris et al. (2008).

The IF hybrid combines IF outputs of the two mixers, such that the IF signal appears at one output and is then amplified by two cryogenic 2-4 GHz IF amplifiers connected in series and cooled to respective temperatures of $4 \mathrm{~K}$ and $12 \mathrm{~K}$. The amplitude component of the sideband LO noise is directed to the other port of the IF hybrid and terminated. After cryogenic amplification, the IF output from the receiver passes through an upconverter. It converts the IF band of $2-4 \mathrm{GHz}$ specified for this mixer band to a 7-5 GHz band and thereby, centers all SHFI IF bands to $6 \mathrm{GHz}$.

Superconducting HEB mixers are based on ultrathithin 4-5 nm thick $\mathrm{NbN}$ film and fabricated on two separated crystalline quartz substrates of dimensions $1000 \mu \mathrm{m} \times$ $70 \mu \mathrm{m} \times 17 \mu \mathrm{m}$. The mixers have to be similar in characteristics to maximize the rejection of the LO noise. Mixer chips fit into substrate channels with suspended microstrip channels fabricated using a previously developed micro-machining technique (Pavolotsky et al. 2005; Desmaris et al. 2008). Finally, the mixers and other parts of the receiver are mounted on a compact mixer-assembly (Fig. 8), which is attached to the $4 \mathrm{~K}$ cold plate inside the facility dewar.

The noise temperature across the RF band was verified after installing the dewar at the telescope using the facility calibration unit. Figure 9 shows the DSB noise temperature averaged over 2-4 GHz (7-5 GHz up-converted).

\section{On-sky tests}

The text below presents a summary of initial astronomical observations performed with SHFI during the on-sky commissioning March-April 2008.

\subsection{Stability}

After installation on the telescope, the stability of all bands was analyzed at different $\mathrm{LO}$ frequencies. The facility backend was used to collect the IF output power at each (1 MHz wide) channel, while an absorber was used to blind the RF window. The

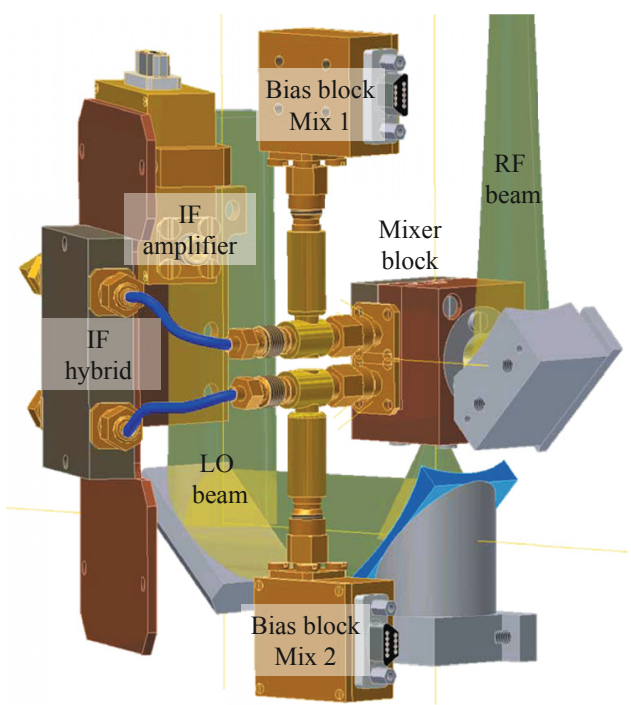

Fig. 8. Band T2 mixer assembly.

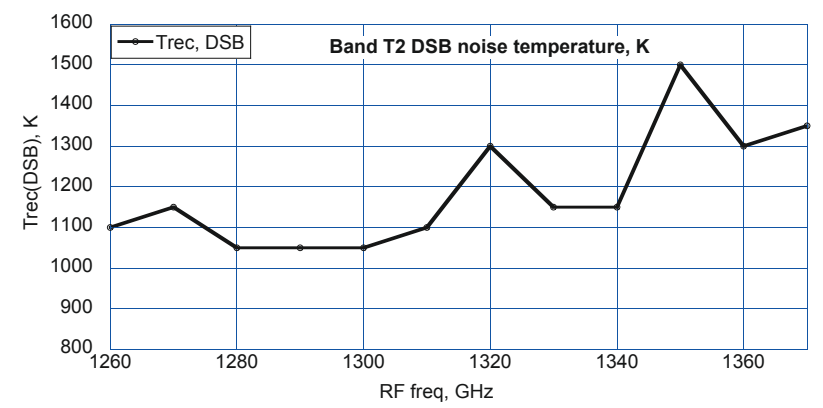

Fig. 9. Noise temperatures of band $\mathrm{T} 2$ across RF measured with the facility calibration unit and for LSB tuning.

total power Allan variance plots for two of the channels and all bands are shown in Fig. 10, where the variance was normalized to the square of the mean value of the channel data set. The measured Allan time is $\geq 70$ s for any of the bands.

In the context of spectroscopic observations, the accurate measurement of the continuum level is not as important as the flatness of the baseline that can be disturbed by fluctuations, which are not common to all the channels. Therefore, we also analyzed the noise produced from taking the difference between two of the backend channels (gain-corrected), where common instabilities such as for example the gain drift of the system were removed from the statistics of the measurement (Schieder et al. 1989 , 2001). The resulting spectroscopic Allan time is $\geq 100 \mathrm{~s}$ in any band. The total power Allan time was also measured over $1 \mathrm{GHz}$ of bandwidth and is of the order of 3-4 s for bands 1 and 2 ( $1 \mathrm{GHz}$ total power stability was not measured for the T2 band).

The T2 band stability demonstrates a total power (over $1 \mathrm{MHz}$ bandwidth) Allan time of more than 30-35 s performed at the $1267 \mathrm{GHz}$ RF frequency, whereas the spectroscopic Allan time is better than $100 \mathrm{~s}$. Measurements performed at a RF frequency of $1382 \mathrm{GHz}$ provide Allan times of $70 \mathrm{~s}$ and $200 \mathrm{~s}$ in the case of total power and spectroscopic Allan variance, respectively (Fig. 10). The measured stability results for the T2 band are fully compatible with the stability of SIS mixers for the other SHFI bands. To our knowledge, these stability values are also the best ever reported for HEB mixers. We attribute this improvement to the implementation of the balance scheme, which reduces effects of the LO instability (Meledin et al. 2008). 

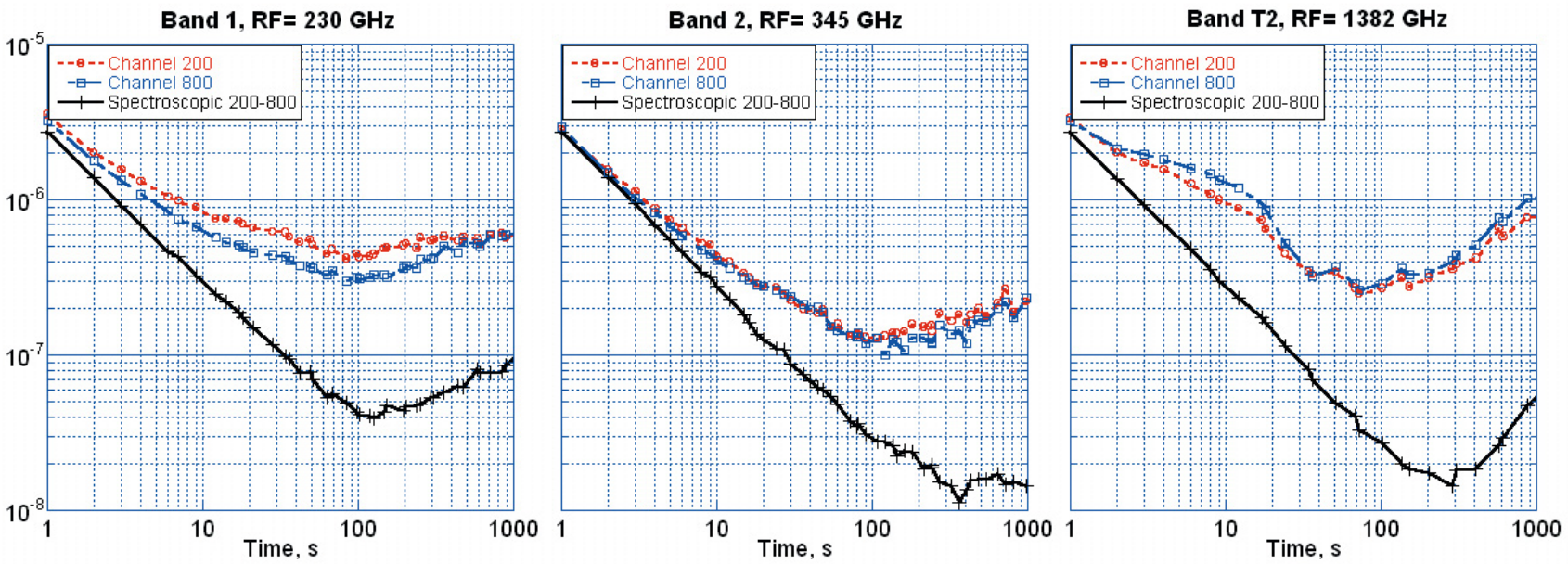

Fig. 10. The normalized $\left(\sigma(\tau)^{2} / \mu^{2}\right)$ total power Allan variance vs. integration time over $1 \mathrm{MHz}$ bandwidth for channels 200 and 800 of the backend, and the corresponding spectroscopic Allan variance.

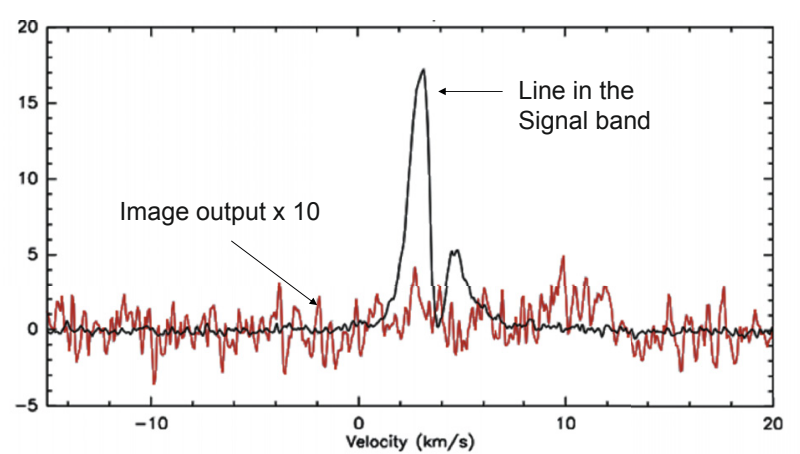

Fig. 11. HCO + (3-2) line in IRAS 16293; LO is set to be $261.5 \mathrm{GHz}$; the black curve shows the line $(267.5 \mathrm{GHz})$ in the USB. The red curve shows the image (LSB) output of the mixer multiplied by 10 . The center of the velocity axis is aligned with the center of the IF band.

\subsection{Image band cancelation}

To verify the sideband-separating properties of bands 1 and 2 , a number of sky sources were observed at both signal and image outputs of the mixer. In Fig. 11, IRAS 16293 was tuned to $\mathrm{HCO}+(3-2)$ in the USB (black curve). The red curve shows the line at the LSB output of the mixer, where Y scale has been multiplied by 10 ; the line is suppressed in the image output by $15 \mathrm{~dB}$.

A number of lines were tuned in both sidebands to enable a comparison of their intensities. Figure 12 shows the $330.59 \mathrm{GHz}$ ${ }^{13} \mathrm{CO}(3-2)$ line tuned to the USB, $(\mathrm{LO}=324.58 \mathrm{GHz})$ with a black curve; the blue curve shows the spectrum at the image (LSB) band multiplied by 10 . The same line was then tuned to the LSB $(\mathrm{LO}=336.59 \mathrm{GHz})$ represented by red curve; the green curve shows the spectrum at the image (USB) band multiplied by 10 .

From Fig. 12, we can see that changing the signal sideband of the mixer does not change substantially the line intensity and profile; the line is suppressed in the image band by $\sim 14 \mathrm{~dB}$.

No laboratory or on-sky measurements were performed to estimate the ratio of sideband gains for the T2 band. Earlier onsky measurements performed by Risacher et al. (2006a) with the APEX 2A DSB receiver were possible due to the existence of a pair of OCS lines with rotational transitions spaced by $\approx 12 \mathrm{GHz}$.

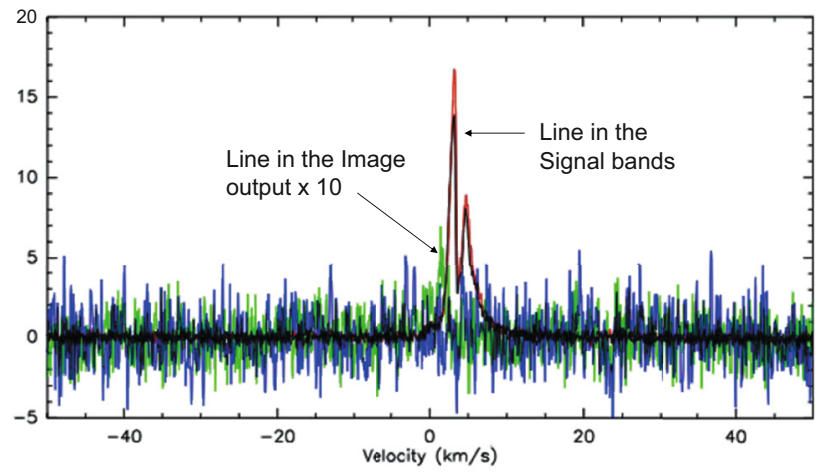

Fig. 12. Line intensities observed with different sidebands of Band 2. IRAS 16293 tuned to ${ }^{13} \mathrm{CO}(3-2)$ in the USB is indicated by the black curve and in the LSB by the red curve. The plot also shows spectra at the respective image outputs of the mixer, where the $\mathrm{Y}$ scale has been multiplied by 10 as blue and green curves.

The sideband gain ratio in this case was found to be close to one with an estimated accuracy of $15 \%$.

\subsection{Beam shape, efficiencies, and pointing}

To verify the band 1 beam quality, a $15 \mathrm{~min}$ on-the-fly (OTF) map of Mars (Fig. 13) was taken over IF bandwidth of $1 \mathrm{GHz}$. The right plot of Fig. 13 is an attempt to reveal sidelobes in the beam, which are not visible to the level of the available signalto-noise ratio.

Figure 14 shows a ${ }^{12} \mathrm{CO}(3-2)$ line pointing for a circumstellar envelope of the evolved star R-Leo. By using the old APEX2a pointing model, the angular offset corrections were found to be $0.04^{\prime \prime}$ and $1.67^{\prime \prime}$. The offset corrections observed after a series of pointings were found to be less than $\approx 30^{\prime \prime}$ for all of the bands.

Telescope efficiencies were calculated by fitting Gaussian function to continuum cross-scans of planets, where a value of $97 \%$ for the forward efficiency was assumed. The calculated beam and aperture efficiencies, shown in Table 2, are consistent with the expected values (Güsten et al. 2006b). 

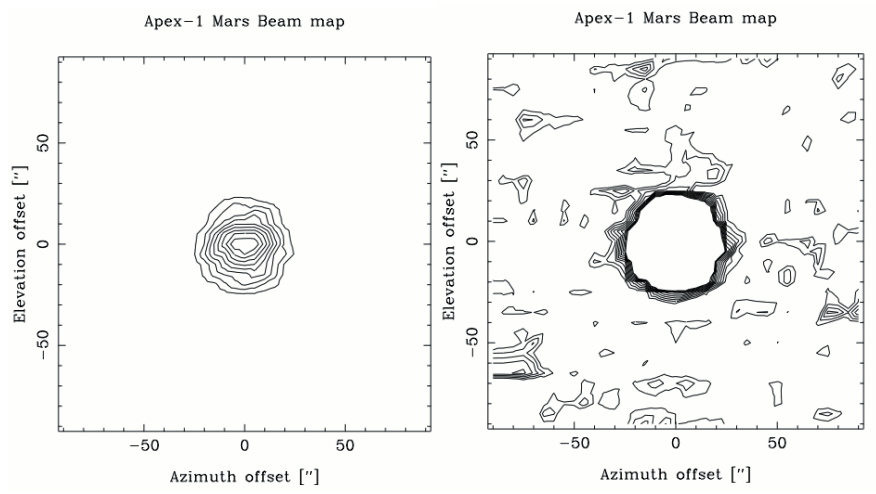

Fig. 13. OTF map on Mars with Band 1. Left: contours starting at $10 \%$ and spaced every $10 \%$. Right: contours starting at $1 \%$ to $10 \%$ and spaced every $1 \%$.

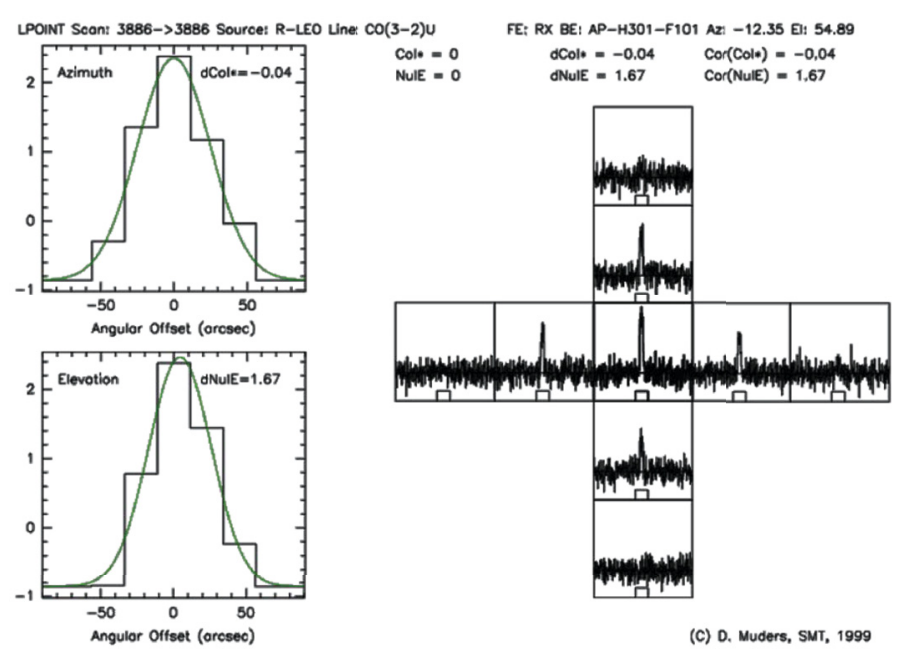

Fig. 14. Example of line pointing with band 2. A ${ }^{12} \mathrm{CO}(3-2)$ cross scan of R-Leo. The angular offset corrections, which correspond to the old APEX-2a pointing model, are $0.04 "$ and $1.67^{\prime \prime}$.

Table 2. Telescope efficiencies with APEX bands 1 and 2.

\begin{tabular}{ccccccc}
\hline \hline $\begin{array}{c}\text { APEX } \\
\text { band }\end{array}$ & Planet & Scan & $\begin{array}{c}\text { Freq. } \\
\text { GHz }\end{array}$ & Elev & $\begin{array}{c}\text { Aperture } \\
\text { efficiency }\end{array}$ & $\begin{array}{c}\text { Beam } \\
\text { efficiency }\end{array}$ \\
\hline 1 & Mars & 2331 & 220 & 38.5 & 0.67 & 0.82 \\
1 & Mars & 2172 & 230 & 41 & 0.66 & 0.81 \\
1 & Mars & 2325 & 230 & 40 & 0.65 & 0.80 \\
1 & Mars & 3795 & 230 & 39 & 0.67 & 0.82 \\
1 & Mars & 2328 & 267 & 39 & 0.59 & 0.72 \\
2 & Jupiter & 2295 & 343 & 39 & 0.59 & 0.72 \\
2 & Jupiter & 2512 & 346 & 39.5 & 0.59 & 0.72 \\
2 & Mars & 3807 & 346 & 36 & 0.53 & 0.65 \\
2 & Mars & 2351 & 356 & 33 & 0.55 & 0.67 \\
\hline
\end{tabular}

\subsection{Observations of narrow line sources}

A few tests were completed to measure narrow-line regions and verify the frequency (velocity) scale. Figure 15 shows the ${ }^{13} \mathrm{CO}(2-1)$ in Barnard 68 dark globule. In terms of both velocity position and line width, the fitted values agree well with observations from CSO (Bergin et al. 2006) and IRAM (Maret et al. 2007) where the corresponding values are $3.31 \mathrm{~km} \mathrm{~s}^{-1}$ and $0.368 \mathrm{~km} \mathrm{~s}^{-1}$, respectively.

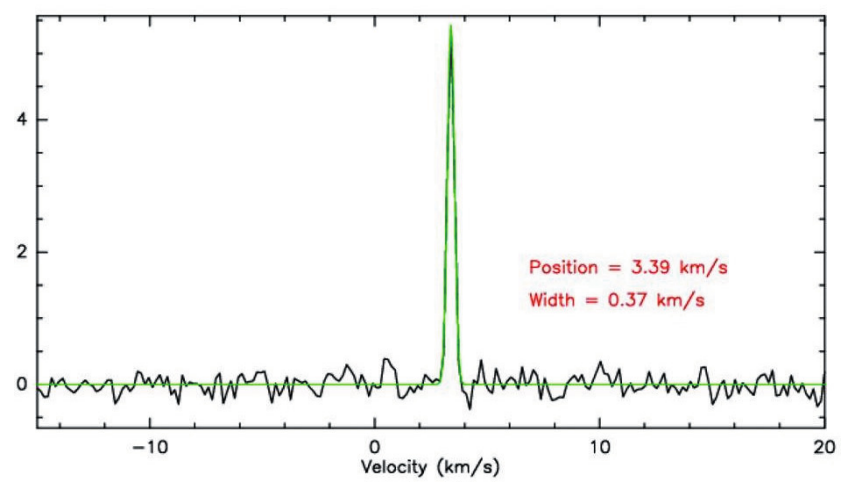

Fig. 15. ${ }^{13} \mathrm{CO}(2-1)$ in the Barnard 68 dark globule. The line position and width agree well with previously published results.

\subsection{Baseline stability}

Good baselines are produced, when using the wobbler to switch between ON and OFF source, usually at a rate of $0.5 \mathrm{~Hz}$, allowing extragalactic observations to be carried out. In positionswitching mode, there is (at least at some frequencies in bands 1 and 2) a standing-wave pattern in the spectrum. Figure 16, shows examples of a broad baseline spectrum taken with band 2, the frequency span of $1.9 \mathrm{GHz}$ is achieved by combining two $1 \mathrm{GHz}$ units of the FFTS.

The spectrum shows the bright $\mathrm{CO} 3-2$ line emission from the luminous, infrared, spiral galaxy IRAS 10567-4310 (ESO 264- G 057). This observation is a part of a larger project (Hurley et al.) to study the correlation of molecular gas properties with the occurrence of AGN in a distance-limited and IR-selected sample of galaxies known as COLA (Corbett et al. 2002).

Figure 17 is another example spectrum taken with the $\mathrm{T} 2$ band that shows a $\mathrm{CO}(11-10)$ line in IRC +10216 (CW-Leo), the brightest asymptotic giant branch star.

\section{Conclusions}

We have presented results from the sky commissioning of the Swedish Heterodyne Facility Instrument installed at the APEX telescope in March-April 2008. The instrument presently contains two SIS sideband-separating mixers (band 1 and 2) and one balanced HEB mixer (T2). APEX bands 1 and 2 are 2SB mixers operating in SSB mode (only one sideband is available at the time). This mode of operation is preferred since replacing one of the IF amplifiers with a matched load provides better match to the IF hybrid port, producing better rejection ratios.

The typical SSB noise temperatures measured at the telescope using the facility calibration unit was between 100 and $300 \mathrm{~K}$ for bands 1 and 2 . The sideband-rejection ratios were measured after the installation of the instrument on the telescope with CW signal and the same test procedure (Kerr et al. 2001) as used in the laboratory measurements. By using on-sky sources, the line suppression in the image band was found to be better than for measurements either in the laboratory or at the telescope using $\mathrm{CW}$ signal. The reason for this difference was either saturation caused by the rich spectral content of the $\mathrm{CW}$ source (a harmonic mixer pumped at $\approx 14 \mathrm{GHz}$ ), or is related to mismatch between the $\mathrm{CW}$ source and the RF optics. This issue needs further investigation. The lowest suppression observed using $13 \mathrm{CO}(2-1)$ line at $220.39 \mathrm{GHz}$ was of the order of $13 \mathrm{~dB}$. 


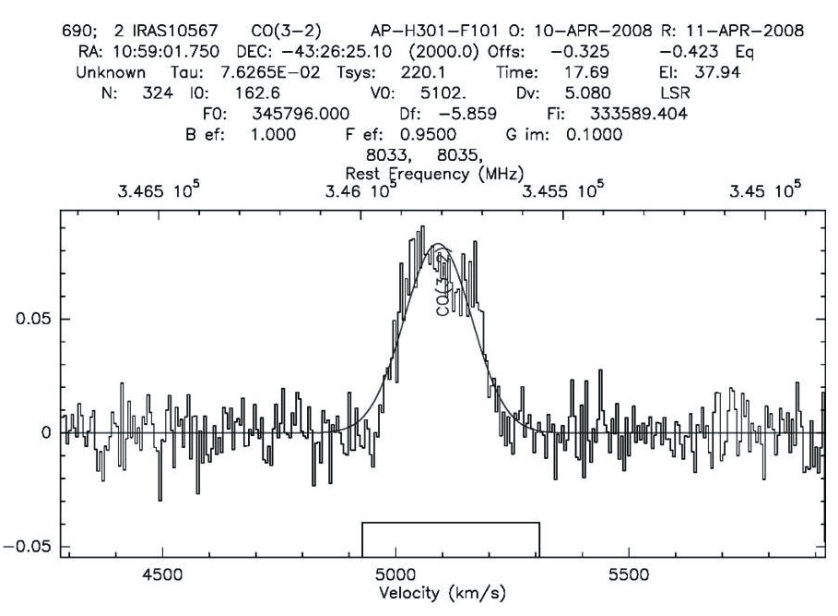

Fig. 16. $\mathrm{CO}(3-2)$ observation from IRAS 10567 taken with APEX-2; no correction to the baseline has been applied other than a polynomial of order 0 . The total observation time was about $36 \mathrm{~min}(\approx 18 \mathrm{~min}$ on each of the ON/OFF positions). With permission from Hurley.

$\mathrm{CO}(11-10) @ 1267 \mathrm{GHz}$ in CW-LEO

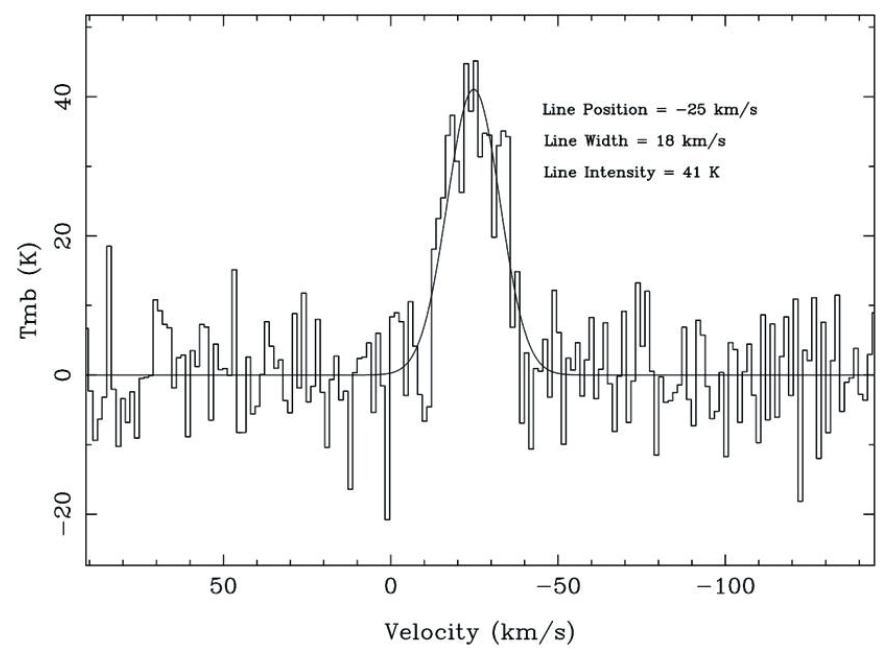

Fig. 17. $C O(11-10)$ observation from IRC +10216 taken with APEX-T2.

Due to lack of power in the existing $\mathrm{THz}$ sources, we used a waveguide balanced mixer configuration for the T2 band. The mixer provides a DSB noise temperature of the order of $1200 \mathrm{~K}$. It also demonstrates remarkable stability times i.e. the total power stability over the $1 \mathrm{MHz}$ bandwidth is better than $30 \mathrm{~s}$, whereas the spectroscopic (difference of two channels $0.6 \mathrm{GHz}$ apart) Allan time is of the order of $200 \mathrm{~s}$. We believe that the excellent stability of this band is due to the use of a balance scheme that reduces the effects of the LO variations.

Each mixer assembly in the cryostat is temperature stabilized reducing the influence of the temperature variations due to the cold-head cycle. This, in combination with temperaturestabilized LO sources, reduces the switching time between the bands. The time required to derive stable mixer performance when switching to another band is of the order of three minutes.

One result of the commissioning is the recommendation of a sideband for each sky frequency. The user interface that controls the receiver provides the possibility to change easily to the sideband that provides lower noise and better rejection ratios. For example, there is a region of $\mathrm{LO}$ frequencies in band 2 where the rejection ratio is poor due to a mechanical problem in the LO waveguide coupler. The receiver can still provide good performance by avoiding these LO frequencies i.e. by selecting an appropriate sideband below or above the bad region.

Acknowledgements. Authors would like to thank R. Hurley and S. Aalto for the help with the data regarding the COLA sample (Fig. 16). Thanks to Lars-Göran Gunnarsson, Leif Helldner, Karl-Åke Johansson and Bhushan Bilade for their assistance in building the hardware. This work is funded by the Swedish Research Council and the Wallenberg Foundation by their respective grants. Additional support is provided by EU FP6 AMSTAR program.

\section{References}

Belitsky, V., Lapkin, I., Monje, R., et al. 2006, Millimeter and Submillimeter Detectors for Astronomy III, Proc. SPIE, 6275

Belitsky, V., Lapkin, I., Vassilev, V., et al. 2007, Infrared and Millimeter Waves Bergin, E. A., Maret, S., van der Tak, F. F. S., et al. 2006, ApJ, 645, 369

Corbett, E. A., Norris, R. P., Heisler, C. A., et al. 2002, ApJ, 564, 650

Desmaris, V., Meledin, D., Pavolotsky, A., Monje, R., \& Belitsky, V. 2008, J. Micromech. Microeng., 18

Güsten, R., Nyman, L. A., Schilke, P., et al. 2006a, A\&A, 454, L13

Güsten, R., Booth, R. S., Cesarsky, C., et al. 2006b, Proc. SPIE, 6267, 626714

Hurley, R., Conway, J., Aalto, S., et al. 2008, in prep.

Kerr, A., \& Pan, S.-K. 1996, Proc. Seventh Int. Symp. Space Terahertz Technology, Charlottesville, 207

Kerr, A., Pan, S.-K., \& Effland, J. 2001, ALMA Memo, 357

Lapkin, I., Nyström, O., Belitsky, V., et al. 2008, Proc. of the 19th International Symposium on Space Terahertz Technology

Maas, S. 1993, Microwave mixers, Artech House

Maret, S., Bergin, E., \& Lada, C. 2007, ApJ, 670, L25

Meledin, D., Pantaleev, M., Pavolotsky, A., et al. 2006, 16th International Symposium on Space Terahertz Technology

Meledin, D., Pavolotsky, A., Desmaris, V., et al. 2008, IEEE Microwave Theory and Technique, submitted

Monje, R., Vassilev, V., Belitsky, V., et al. 2004, 12th International Conference on Terahertz Electronics, 699

Monje, R., Belitsky, V., \& Vassilev, V. 2006, Microwave Symposium Digest, IEEE MTT-S International, 1169

Ossenkopf, V. 2008, A\&A, 479, 915

Pavolotsky, A., Meledin, D., Risacher, C., et al. 2005, Microelectronics J., 36, 683

Pineda, J. L., \& Bensch, F. 2007, A\&A, 470, 615

Risacher, C., \& Belitsky, V. 2003, IEEE Microwave and Wireless Components Letters, 13, 96

Risacher, C., Monje, R., Vassilev, V., et al. 2006a, Proc. SPIE, 6275

Risacher, C., Vassilev, V., Monje, R., et al. 2006b, A\&A, 454, L17

Schieder, R., \& Kramer, C. 2001, A\&A, 373, 746

Schieder, R., Tolls, V., \& Winnewisser, G. 1989, Exp. Astron., 1, 101

Vassilev, V., Belitsky, V., Urbain, D., \& Kovtonyuk, S. 2001, IEEE Microwave and Wireless Components Letters, 11, 30

Vassilev, V., Henke, D., Lapkin, I., et al. 2008, IEEE Microwave and Wireless Components Letters, 18, 58 